\title{
EL CAMBIO EN LA EDUCACIÓN: VIEJAS RAÍCES, NUEVAS PRÁCTICAS DOCENTES MEDIADAS POR TIC*
}

\section{Pablo César Hernández Cerrito ${ }^{I}$}

\section{Francisco Javier Mancilla Venegas ${ }^{2}$}

\section{Resumen}

El cambio en la educación es un desafío complejo, requiere de la participación de diversos actores, de procesos a corto y a largo plazo para transformar la forma de enseñar. En el camino al cambio, encontramos obstáculos, resistencias y tradiciones profundas. En esta línea de pensamiento, el desarrollo del trabajo colaborativo empleando el potencial de las Tecnologías de la Información y la Comunicación (TIC), promete trascender de la zona de confort hacia una zona de innovación. Insertar nuevas prácticas docentes mediante TIC en viejas raíces, es una oportunidad que tienen las Instituciones de Educación Superior (IES) para construir comunidades de conocimiento y aprendizaje aprovechando el potencial pedagógico del binomio docencia-investigación e investigación-docencia.

El propósito de esta memoria es analizar, reflexionar y mostrar cómo los docentes son líderes que diseñan el futuro educativo y juegan un papel preponderante para enfrentar el cambio. Cómo la estructura y cultura organizacional influyen en la formación de los líderes, pero aún más los líderes influyen en el cambio y en la transformación de la organización. En este sentido, se propone un plan de gestión del cambio transformacional, donde se deja ver que la voluntad, la ética, la mentalidad innovadora y la responsabilidad social por la docencia de calidad, son componentes indispensables para repensar, ejercer y vivir el liderazgo que trasciende.

1 Profesor-Investigador virtu@mi, UAM-Iztapalapa.

2 Profesor-Investigador del Departamento de Economía de la UAM-Iztapalapa

* Fecha de recepción: 20/09/2018. Fecha de aceptación: 29/07/2019. 
Palabras clave: Cultura Organizacional, Nuevas prácticas docentes con TIC, Liderazgo e Innovación educativa.

\section{Abstract}

The change in education is a complex challenge, it requires the participation of diverse actors, of short and long term processes to transform the way of teaching. On the road to change, we find obstacles, resistances and deep traditions. In this line of thought, the development of cooperative work using the potential of Information and Communication Technologies (ICT), promises to transcend the comfort zone towards an area of innovation. Insert new teaching practices through ICT in old roots, is an opportunity for higher education institutions to build knowledge and learning communities taking advantage of the pedagogical potential of the binomial teaching-research and research-teaching.

The purpose of this report is to analyze, reflect and show how teachers are the leaders who design the educational future and the preponderant role to face change. Organizational structure and culture influence the formation of leaders, but even more leaders influence the change and transformation of the organization. In this sense, a transformational change management plan is proposed, where the will, ethics, innovative mentality and social responsibility for the teaching of quality, the indispensable components for rethinking, the direction and the leadership that transcends.

Keywords: Organizational culture, New teaching practices with ICT, Leadership and Educational Innovation.

\section{Introducción}

El reto generacional se viene manifestando desde hace más de veinticinco años a partir del surgimiento y uso de las TIC, situación que ha transformado el enfoque formativo en la educación superior. La nueva forma de educar requiere una integración efectiva de las tecnologías en el contexto actual de la enseñanza con el fin de proporcionar a los estudiantes habilidades que se apliquen en diversos contextos disciplinarios. 
Tradicionalmente, la educación solía ser una cuestión de transferencia unidireccional de información: del docente al estudiante. El método ex cáthedra, donde el docente se expresa como la única autoridad legítima, era el método común. Hay nociones de que los estudiantes deben estar capacitados para aprender de manera más autónoma y, para acceder y asimilar la información de forma más independiente, lo que podría proporcionar un pilar para el aprendizaje permanente. Los nuevos modelos pedagógicos que incluyen las TIC deberían proporcionar a los estudiantes más rezagados apoyo complementario para reducir las diferencias en el nivel de aprendizaje al ingreso a la universidad. Enseñar a los estudiantes a buscar información, procesar datos y presentar información; colaborando entre estudiantes del mismo campus o de campus similares, así como para enseñar a los estudiantes cómo controlar su propio progreso de aprendizaje y mejorar su conocimiento a lo largo de su trayecto formativo. En esta dirección, la función principal de los profesores-investigadores será actuar como expertos y líderes para motivar el aprendizaje (Djukic, Mrdjenovic y Jokovic, 2015).

Por lo señalado, este trabajo, se centra en tres puntos sustantivos que abordan la integración de las TIC en la práctica docente en la educación superior; 1) tiempos de cambio en los procesos de enseñanza aprendizaje, 2) binomio docencia investigación, investigación docencia como palanca de cambio y 3 ) anclar nuevas prácticas docentes mediante TIC en viejas raíces.

\section{Tiempos de cambio en los procesos de enseñanza aprendizaje}

Vivimos en tiempos de cambio. Cambios en la gestión, en la pedagogía, en los procesos de enseñanza aprendizaje. Estos cambios implican múltiples desafíos. Uno de los desafíos más importantes, es el cambio de paradigma donde el rol del profesor y el rol del estudiante se transformen; el profesor como un facilitador y guía, y el estudiante como un sujeto activo con habilidades de pensamiento reflexivo y crítico para la solución de problemas de su entorno. 
En las Instituciones de Educación Superior (IES) con arraigo en modelos educativos tradicionales y profesores acostumbrados a la clase por cátedra y por exposición, donde el estudiante asume un rol pasivo, será un reto y desafío constante el desarrollo del liderazgo de transformación. De acuerdo con Bass, el liderazgo transformador deberá incluir una visión de futuro, inspirar altas expectativas y proporcionar nuevas ideas y enfoques. En aportación de Leithwood, el ejercicio del liderazgo en la educación deberá contener una visión compartida, apoyo individual y un estímulo intelectual, descentralización de responsabilidades y autonomía de los profesores para promover una cultura propia y colaborativa (como se citó en Salazar, 2006).

En este sentido, es de trascendencia darle la palabra y el poder a los estudiantes, que ellos sean los actores estelares en la integración de la nueva escuela y la educación del futuro. Lo antes mencionado, trae consigo cambios y, de manera natural, resistencias, al cambio mismo, y a mantener el status quo. La educación de alta calidad, requiere de un liderazgo colaborativo para enfrentar los desafíos y garantizar un mejor futuro. Al respecto, consideramos que los pilares de la educación son resultado de un esfuerzo de cooperación (Johnson \& Johnson, 2015) ${ }^{3}$.

Una educación más abierta, más flexible a las necesidades del estudiante. Un cambio de paradigma conlleva a cambios profundos en la organización social del trabajo, en el liderazgo educativo y en la forma de enseñar y aprender; un cambio en la forma en cómo concebimos la educación del futuro: ¿Por qué educamos? reto que exige un cambio de creencias y prácticas docentes, ¿Para qué educamos? para formar sujetos críticos con habilidades para solucionar problemas de su entorno ¿Con qué medios y tecnología educamos? utilizamos tecnologías

3 De acuerdo con el Instituto de Aprendizaje Cooperativo fundado por Johnson \& Johnson, cooperación es trabajar juntos para lograr metas compartidas. Dentro de las situaciones de cooperación, los individuos buscan resultados, beneficios para ellos y para todos los miembros del grupo. El aprendizaje cooperativo es el uso didáctico de grupos para que los alumnos trabajen juntos y así maximizar su propio aprendizaje y el de los demás (Johnson \& Johnson, 2015). 
emergentes y metodologías innovadoras para un aprendizaje significativo y duradero.

De acuerdo con Lueny Morell (2010) uno de los retos primordiales de la enseñanza profesional en el siglo XXI radica en comprender al estudiante contemporáneo: creativo, móvil, multi-tarea, colaborativo y productor (como se citó en Corporación Digital Colombiana, 2012).

En el contexto institucional de la Universidad Autónoma Metropolitana Unidad Iztapalapa (UAM-I), algunas de las necesidades apremiantes para la formación del trabajo cooperativo entre docentes y mayor participación de los estudiantes que se proponen son:

- Visión compartida e incluyente en la mejora de la calidad de enseñanza aprendizaje.

- Integración de metodologías de trabajo colaborativo e inclusión de estilos de aprendizaje y enseñanza dentro y fuera del aula de clases.

- Abrazar a la tecnología como herramienta de innovación educativa mediante el desarrollo de competencias y habilidades digitales.

- Formación y actualización docente en habilidades comunicativas y pedagógicas mediante TIC.

- Formación y actualización de estudiantes en habilidades de lecto-escritura mediante TIC.

- Fomentar la identidad académica de docentes y de estudiantes a través de redes presenciales y virtuales.

De acuerdo con Barber y Mourshed (2008) la planta docente altamente capacitada en aspectos relacionados con las estrategias didácticas en comunicación, trabajo en equipo, evaluación y liderazgo educativo incide directamente en los resultados académicos de los estudiantes. Sumando al aporte de Barber y Mourshed (2008), consideramos pertinente, en el contexto institucional de la universidad, que tanto la docencia como la investigación interactúen de manera dinámica e 
integral en los procesos educativos tanto para fortalecer la enseñanza como el aprendizaje de la comunidad académica.

\section{Binomio docencia investigación, investigación docencia como palanca de cambio}

Para Morán (2004), el docente investigador es el que enseña lo que investiga; hace de su práctica docente el objeto de estudio con ingenio, creatividad y compromiso. En esta línea de pensamiento, se propone la investigación para la docencia; análisis de la práctica docente y la investigación como docencia; inducir al estudiante al quehacer de indagación y descubrimiento. En los procesos de enseñanza aprendizaje, los estudiantes son considerados como investigadores (Moran, 2004).

De acuerdo con Sancho (2001), existen factores que favorecen transferencias positivas entre ambas actividades, docencia investigación e investigación docencia. Las transferencias positivas son:

- Los académicos no pueden ser buenos sin hacer investigación, aunque un buen investigador puede ser un pésimo docente.

- Algunas de las infraestructuras conseguidas a través de proyectos de investigación también se utilizan en actividades de enseñanza.

- Las actividades de investigación contribuyen a poner al día el currículum, afectando de forma positiva a los cursos especializados.

- Si los cursos se relacionan con el perfil investigador del profesorado, la relación es favorable (Sancho, 2001).

Para Ustaritz (2003), el problema en la falta de integración de la investigación con la docencia es un problema estructural, institucional y pedagógico. El autor propone, orientar la enseñanza de la investigación científica desde un modo cooperativo -lograr una integración real entre el maestro y el alumno--. Una socialización científica donde se destaque en el proceso de transmisión del conocimiento, el de tipo tangible; formación teórica y de las habilidades, y el de tipo tácito; enseñar el saber hacer, el gusto por la ciencia. Es una nueva enseñanza de la investigación con la enseñanza del oficio. 
Es necesario [...] que la investigación tenga un impacto más envolvente y determinante en la enseñanza, como nutriente del currículo y orientador de prácticas docentes; así como en las instancias de formación y actualización de profesores. Uno de estos retos tiene que ver con la capacidad de vislumbrar las estrategias de lucha política y académica contra las fuerzas que se resisten al cambio y las innovaciones; lo cual implica la revaloración y reorientación de la investigación (Ustaritz, 2003:170).

Sumado a las aportaciones de Sancho (2001) y de Ustaritz (2003), un área de oportunidad para potenciar la interacción docencia investigación e investigación docencia en la vida académica y organizacional, es el uso pedagógico de las TIC. En esta misma idea, las TIC pueden convertirse en una herramienta de transformación y de innovación. El uso ético y crítico de las TIC puede mejorar la ciencia, la cultura y la educación de una sociedad (Hernández, 2015).

Por consiguiente, la propuesta de cambio que consideramos en el contexto y en las circunstancias institucionales que se pueden llevar a cabo con liderazgo en diferentes instancias a nivel colectivo son:

1. Promover en las políticas de docencia y en las estructuras organizacionales de evaluación el mismo reconocimiento que se le otorga a la investigación. Ambas actividades son sustantivas para el desarrollo de la universidad. La contratación laboral en nuestra universidad es como profesor-investigador por lo que es pertinente tengan el mismo valor y reconocimiento académico.

2. Diseñar e implementar un perfil de ingreso para la docencia investigación universitaria con las características, capacidades, valores y competencias acordes a la identidad, compromiso y valores institucionales.

3. La formación docente investigador obligatoria en la incorporación de nuevas generaciones de profesores.

4. Implementar un programa de formación sistemático con seguimiento y asesoría permanente para aprovechar el potencial de las TIC tanto en la docencia como en la investigación. 
5. La construcción de Comunidades Profesionales de Aprendizaje con interés en Investigación Educativa.

6. En conjunto con el gobierno y la universidad establecer acuerdos para la formación sistemática y de alta calidad, respetando la autonomía, la libertad de cátedra y el desarrollo de investigación científica innovadora con ética profesional.

De acuerdo con Bates y Sangrá (2012), la formación sistemática para todos los profesores es fundamental no sólo para aprovechar el uso de las TIC, sino para mejorar la enseñanza de manera eficaz. Bates y Sangrá afirman que existe gran resistencia al cambio por parte de la cultura organizativa dominante y tradicional, reto trascendente a enfrentar. A nivel individual, los cambios tendrían que buscar el desarrollo de la conciencia, la recuperación del arte y oficio de ser docente. La ética, la razón y la emoción como parte fundamental de la unión docencia investigación.

\section{Anclar nuevas prácticas docentes mediante TIC en viejas raíces}

El cambio es un desafío continuo, requiere de diferentes procesos y actores para transformar la cultura organizacional. En el camino al cambio, encontramos obstáculos, resistencias y tradiciones profundas. Sin embargo, el desarrollo de liderazgo mediante un plan de buenas prácticas aprendidas de experiencias de éxito, augura trascender el lugar común de la zona de confort y complacencia.

El liderazgo que se ejerza en una comunidad que aprende será fundamental para encauzar la dirección, promover confianza, respeto, responsabilidad, comunicación y colaboración. Así como para motivar y crear las condiciones necesarias para gestar las transformaciones. La mente creativa y el pensamiento crítico para la solución de problemas al enfrentar el cambio serán herramientas imprescindibles.

En el entendido que no hay cambios que perduren sin un liderazgo que trascienda. En el presente apartado, se desarrolla un plan de 
gestión del cambio transformacional con las ocho fases que Kotter (2007) propone:

1. Imprimir el carácter de urgencia.

2. Crear una coalición.

3. Desarrollar la visión y la estrategia.

4. Comunicar la visión del cambio.

5. Capacitar la acción.

6. Generar éxitos a corto plazo.

7. Consolidar los logros y generar más cambio.

8. Anclar los nuevos puntos de vista a la cultura.

De acuerdo con Kotter (2007), el primer paso del proceso para generar el cambio organizacional es, 1) imprimir el carácter de urgencia; analizar las realidades competitivas e identificar las crisis potenciales y grandes oportunidades. En este sentido, una fortaleza importante en el contexto de estudio UAM-Unidad Iztapalapa, es contar con profesores con alto grado de formación científica y académica. De acuerdo con el anuario estadístico de 2017 , el $70.8 \%$ de la planta académica tiene grado de doctor, $20.3 \%$ de Maestría y más del 55\% pertenece al Sistema Nacional de Investigadores (SNI). No obstante, una de las debilidades de esta casa de estudios, por un lado, es la falta de producción científica de las propias prácticas pedagógicas. Por otra parte, un porcentaje significativo de los profesores tiene una edad promedio de más de 60 años, muestran carencias en el desarrollo de habilidades pedagógicas para el manejo óptimo de las tecnologías emergentes.

Una de las oportunidades al respecto, es mejorar la calidad de la enseñanza y el aprendizaje de los alumnos mediante investigación educativa y el aprovechamiento pedagógico y eficiente de las TIC. En el apartado de Políticas de Docencia, la Legislación Universitaria de la UAM menciona que "la docencia se fortalezca mediante la actualización disciplinaria del personal académico y con la incorporación de los avan- 
ces pedagógicos y tecnológicos que hagan más eficiente y participativo el proceso de enseñanza aprendizaje" (UAM, 2003: artículo 2.10).

La amenaza urgente en la universidad es la tendencia descendente de eficiencia terminal; deserción, bajos promedios y aprovechamiento escolar deficiente. La eficiencia terminal de la UAM paso del 2006 de $53.8 \%$ al 2013 a 24.13\% (UAM, anuario estadístico, 2017). En universidades públicas pares, la eficiencia terminal es de mayores porcentajes (La Jornada, 2014).

\section{Gráfica 1. Eficiencia terminal en la UAM. Información del trimestre 2017-O (Archivo general de alumnos de la $1^{\text {a }}$ semana de $\left.2018-I\right)$}

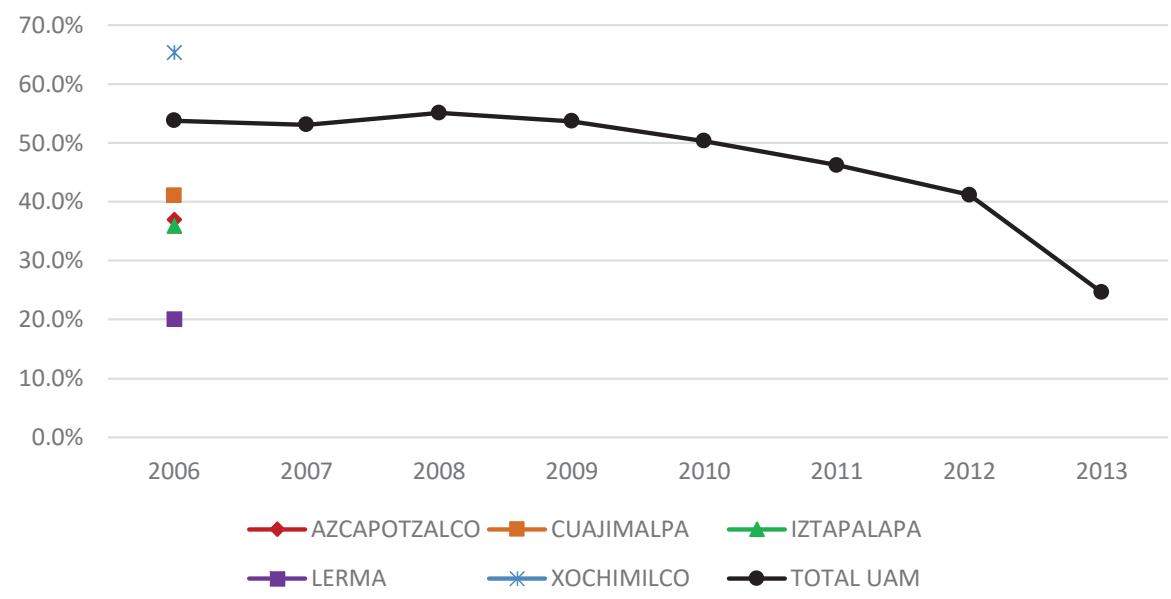

Fuente: Elaboración propia a partir del Anuario estadístico 2017 (Universidad Autónoma Metropolitana, 2017: 114).

En la segunda fase, 2) crear una coalición para promover el cambio, es deseable se abran espacios de discusión tanto para profesores de la UAM como para profesores expertos de otras universidades e interesados en la mejora educativa de la institución, para analizar, discutir y generar propuestas. Como resultado de los espacios de discusión, es recomendable crear grupos de trabajo cooperativo según intereses. De acuerdo al perfil de intereses académicos se puede desarrollar un seminario per- 
manente con objetivos y metas generadas por el propio grupo que permitan brindar propuestas de solución a la problemática educativa. De acuerdo con las propuestas e ideas colectivas que deriven del seminario, será posible elaborar la etapa 3) visión y las estrategias de cambio.

En la etapa 4) comunicar la visión de cambio, por medios impresos y digitales se deberá comunicar a la comunidad académica de las propuestas. Lo más importante en esta etapa, será que los grupos lleven a cabo las propuestas en sus distintas áreas de conocimiento para mostrar los resultados, aquellos proyectos con resultados favorables se pueden publicar como experiencias de éxito educativo.

Para la etapa 5) capacitar para la acción, los profesores con casos de éxito de buenas prácticas educativas y uso eficiente de las TIC, pueden organizar un taller para capacitar a los docentes en el desarrollo de metodologías didácticas innovadoras en el aula. Uno de los grupos de mayor importancia para impulsar el cambio, tendrán que ser los profesores más motivados y los profesores de recién ingreso a la universidad. La capacitación se sustentará en las buenas prácticas educativas propuestas en investigaciones de Chickering y Gamson (1987):

1. Promover el contacto entre estudiantes y profesores.

2. Desarrollar la reciprocidad y la cooperación entre los estudiantes.

3. Fomentar el aprendizaje activo.

4. Dar realimentación rápida.

5. Hacer hincapié en el tiempo dedicado a la tarea.

6. Comunicar altas expectativas.

7. Respetar los diversos talentos y formas de aprender.

Los siete principios de buenas prácticas educativas se podrán potenciar con seis fuerzas poderosas en la educación que pueden promover los cambios: la actividad continua, atender las expectativas, la cooperación, la interacción, la diversidad y la responsabilidad (Chickering y Gamson, 1987). En este orden de ideas, el plan de gestión del cambio 
propuesto, se podrá promover en cada una de las ocho fases y en cada fase se podrán promover las fuerzas de cambio de tal manera que se potencien los procesos de transformación.

En la etapa 6) generar éxitos a corto plazo, durante las etapas anteriores, se tendrá que identificar a los profesores más comprometidos, aquellos que muestren ser ejemplo de liderazgo docente y de investigación. Del grupo se podrá conformar una comunidad elite para seguir generando éxitos mediante el cambio de prácticas tradicionales por buenas prácticas docentes e innovaciones pedagógicas con TIC.

De acuerdo con Martínez, Buxarrais y Bara (2002), los profesores universitarios "están abiertos a la innovación, al pensamiento crítico, al progreso y a la búsqueda de rigor y de verdad, pero a la vez son conservadores, cuidan la tradición y no arriesgan en sus estilos de hacer y de ejercer la docencia". Siguiendo con esta línea de pensamiento: "a las universidades y a los universitarios no nos faltan estímulos que nos orienten hacia el cambio; lo que nos falta es voluntad de cambio y garantía de que éste contribuirá a la mejora" (Martínez, Buxarrais y Bara, 2002).

Para Ginés (2004), la necesidad de cambio educativo responde a un cambio intrínseco del modelo pedagógico y un cambio extrínseco del modelo organizativo de las instituciones. Por su parte, el cambio intrínseco, tiene que dirigirse al desarrollo de las habilidades de saber leer y escribir, saber pensar y saber seguir aprendiendo, aprender a relacionarse y a la aplicación de los conocimientos teóricos. Por su parte, el cambio extrínseco, debe estar orientado a brindar flexibilidad para la formación a lo largo de la vida. En este sentido, es deseable que el cambio se consolide y genere un efecto multiplicador.

En la etapa 7) consolidar los logros y generar más cambio, se podrá proponer la creación del primer grupo red multidisciplinario de investigación educativa en la institución. Esta red de investigación para la innovación de los procesos educativos liderará la investigación cientí- 
fica educativa en el campus y fuera del recinto universitario. Estar en la frontera del conocimiento con investigación-acción educativa será un estímulo y motivación para conectar la docencia y la investigación, ambas actividades sustantivas con tradición y presentes en la razón de ser, misión y visión de la universidad.

Entonces, cómo anclar los nuevos puntos de vista en la cultura organizacional dominante, etapa 8; es necesario fijar firmemente las nuevas prácticas en las normas, valores y creencias del grupo. Si las prácticas de cambio son incompatibles con la cultura dominante éstas estarán sujetas a regresión. El reto es insertar las nuevas prácticas en las viejas raíces (Kotter, 2007). Para anclar los nuevos puntos de vista en la cultura universitaria es menester conocer las raíces de la universidad.

Al respecto del conocimiento de la universidad a sus 45 años de existencia; es una universidad que se caracteriza por estar asentada en la tradición (investigación), abierta, interdisciplinaria, autónoma y flexible. Que tiene origen en la cultura náhuatl y significa:

\section{CASA ABIERTA AL TIEMPO "IN CALLI IXCAHUICOPA"}

In calli significa casa en náhuatl Ix (tli) rostro, cáhui (tl) tiempo y -copa hacia, integran ix-cahuicopa, "hacia el tiempo con rostro". El elemento central cáhui (tl) implica "cambio y lo que éste va dejando". En resumen, In calli ixcahuicopa es "casa orientada al tiempo con rostro". Convertida la frase en lema, apunta a los propósitos de la Universidad, que es CASA ABIERTA AL TIEMPO portador de sentido, posibilidad de saber y de diálogo (UAM, Emblema y Lema, 2015).

En el significado y origen de la universidad está arraigado el cambio y los conceptos inherentes para promoverlo. Lograr la conexión docencia investigación mediante la investigación educativa para mostrar los beneficios en la mejora de la docencia, que caracterice a una universidad abierta, interdisciplinaria, autónoma y flexible, sería el anclaje idóneo en la cultura organizacional que facilitaría la permanencia del cambio. 
El promedio de edad de la planta académica de la universidad cada vez es mayor. Poco a poco se están incorporando nuevas generaciones de profesores. En esta idea de recambio generacional, una estrategia para arraigar las nuevas prácticas en viejas raíces es mediante el aprendizaje cooperativo entre pares; profesores con amplia experiencia en investigación motivados por la docencia de calidad enseñando prácticas exitosas a profesores de nuevo ingreso y profesores de nuevo ingreso enseñando a los profesores a utilizar la TIC de manera eficiente para ser aplicados en los procesos de enseñanza aprendizaje.

El concepto de interacción social de Jonh Dewey, el concepto de Zona de Desarrollo Próximo ZDP de Vigostky, donde los más experimentados enseñan a los menos experimentados. La teoría de Wenger sobre las Comunidades de Práctica ${ }^{4}$ para generar un proceso colectivo de aprendizaje y liderazgo interno, serían motores del cambio de las prácticas docentes para anclar los nuevos puntos de vista en la cultura institucional.

\section{Conclusión}

El desafío de cambio de las prácticas docentes tradicionales por prácticas innovadoras, es complejo. Se requiere de un esfuerzo constante, dedicación, paciencia, tolerancia para integrar la diversidad de perfiles, inteligencias e intereses académicos para mirar juntos hacia el mismo horizonte. En este sentido, coincidimos con Paulo Freire al señalar que, la indagación, la búsqueda, la investigación, forman parte de la naturaleza de la práctica docente. Lo que se necesita es que el profesor, en su formación permanente, se perciba y se asuma, por ser profesor, como investigador.

4 Las Comunidades en Práctica son sistemas de auto-organización donde las personas comparten la capacidad de crear y utilizar el conocimiento. Existe una comunidad de práctica, ya que sus miembros producen un proceso colectivo de aprendizaje. Una Comunidad de Práctica es un nodo para el intercambio y la interpretación de la información. El núcleo de una Comunidad en Práctica es la experiencia, su desarrollo depende del liderazgo interno (Wenger, 1998). 
En la tradición universitaria que se sustenta en ser flexible, en la interdisciplina y autonomía, la dialéctica docencia investigación, investigación docencia, puede otorgar identidad al lema de la institución como casa abierta al tiempo. Todo un desafío que implica una transformación en el comportamiento y en la cultura organizacional dominante. Los cambios que conllevan las nuevas tecnologías afectan a la sociedad de forma tan profunda, con tal efecto que, los que no se prevengan para afrontar estos cambios estarán en gran desventaja en un futuro inmediato.

Lo antes mencionado demanda de líderes exitosos, líderes que se adaptan al contexto y a las circunstancias de manera flexible. Líderes que trabajen en equipo y brinden la oportunidad para que las personas desarrollen sus talentos. Será idóneo desarrollar habilidades de pensamiento creativo. El docente como líder, juega un papel preponderante para enfrentar el futuro. La estructura y cultura organizacional influyen en la formación de los líderes, pero aún más los líderes influyen en el cambio y en la transformación de la organización. La mentalidad innovadora y la vocación por la docencia investigación, son componentes indispensables para pensar, ejercer y vivir el liderazgo del cambio. En pocas palabras, un liderazgo, que inspira, transforma y trasciende.

\section{Bibliografía}

Barber, Michael y Mourshed, Mona (2008), Cómo hicieron los sistemas educativos con mayor desempeño en el mundo para alcanzar sus objetivos. Disponible en: http://www.oei.es/pdfs/documento_ preal41.pdf

Bates, Tony y Sangrá, Albert, La gestión de la tecnología en la educación superior. Estrategias par transformar la enseñanza y el aprendizaje, Octaedro, Barcelona, 2012.

Corporación Colombia Digital, Aprender y educar con las tecnologías del siglo XXI, 2012. Disponible en: http://cmap.javeriana.edu.co/servlet/SBReadResourceServlet?rid=1KRS0DP7S-1YWQ573-3CX 
Chickering, Arthur y Gamson, Zelda, Siete Principios de Buenas Prácticas en la Educación, 1987. Disponible en: http://portalacademico. cch.unam.mx/blog/siete_principios

Djukic, A., Mrdjenovic, T., \& Jokovic, J. J., "E-learning and AMRES in teaching architecture”, D. A: Garcia Escudero, \& B. Bardí Milà, edits., III Jornadas sobre Innovación Docente en Arquitectura (JIDA’15) (15), 2015, pp. 90-100.

Ginés, José, "La necesidad del cambio educativo para la sociedad del conocimiento", La Revista Iberoamericana de Educación, núm., 35, 2004, OEI. Disponible en: http://www.rieoei.org/rie35a01.htm

Hernández, Pablo, "El uso de Internet en la Educación”, Painani, el mensajero de la ciencia y las humanidades, núm. 6, febrero - mayo, 2015, UAM, México, pp. 16-17.

Johnson, David \& Johnson, Roger, An Overview Of Cooperative Learning, 2015. Disponible en: http://www.co-operation.org

Kotter, John, Al Frente del Cambio: La Hoja de Ruta del Experto Mundial en Liderazgo del Cambio, Empresa Activa, Ediciones Urano, España, 2007.

La Jornada, "Alcanza el IPN eficiencia terminal de 61\% y $63 \%$ en el nivel medio y superior", 2014. Disponible en: http://www.jornada. unam.mx/2014/08/25/sociedad/041n1soc [22 de septiembre 2015].

Martínez, Miquel, Buxarrais, María y Bara, Francisco, "Ética y formación universitaria" La Revista Iberoamericana de Educación, núm. 29, mayo-agosto 2002, OEI. Disponible en: http://www.rieoei.org/ rie29a01.htm [1 de septiembre de 2015]

Morán, Porfirio, "La docencia como recreación y construcción del conocimiento. Sentido pedagógico de la investigación en el aula", Perfiles Educativos, núm. 106, (26), 2014, pp. 41-72.

Salazar, María, "El liderazgo transformacional ¿Modelo para organizaciones educativas que aprenden?", UNIrevista, núm. 3, (1), 2006, Chile, Universidad de Viña del Mar, pp.1-12. 
Sancho, Juana, "Docencia e investigación en la universidad: una profesión, dos mundos" Educar, núm 28, 2001, pp. 41-60.

UAM, Anuario estadístico (2017), 2017, p. 114. Disponible en: http:// www.transparencia.uam.mx/inforganos/anuarios/anuario2017/ anuario_estadistico2017.pdf

UAM, Legislación Universitaria, Políticas de Docencia, Artículo 2.10. , 2003. Disponible en: http://www.uam.mx/legislacion/

UAM, Emblema y Lema, 2015. Disponible en: http://www.uam.mx/sah/ pre-pa/tema01/identidad.html

Ustaritz, Alejandro, "Docencia e investigación en el aula: una relación imprescindible", en Docencia e investigación en el aula: una relación imprescindible, Centro de Estudios Sobre la Universidad UNAM, México, 2003.

Wenger, Etienne, Communities of practice, learning as a social system, 1998. Disponible en: http://skat.ihmc.us/rid=1227187866819_1140 452997_15052/communities\%20of\%20practice_wenger.doc. 
